\title{
Ethnoveterinary management of cattle helminthiasis among the Fulani and the Mossi (Central Burkina Faso): plants used and modes of use
}

\author{
Adama HILOU $^{1 *}$, Florence RAPPEZ ${ }^{2}$ and Pierre DUEZ ${ }^{2,3}$ \\ ${ }^{1}$ Laboratory of applied Biochemistry and chemistry (LABIOCA), UFR/SVT, \\ Université de Ouagadougou, 09 BP984 Ouaga 09, Burkina Faso. \\ ${ }^{2}$ Laboratory of Pharmacognosy, Bromatology and Human Nutrition, Faculté de Pharmacie, \\ Université Libre de Bruxelles (ULB), CP 205/9, Bd du Triomphe, 1050 Brussels, Belgium. \\ ${ }^{3}$ Department of Therapeutical Chemistry and Pharmacognosy, Faculté de Médecine et de Pharmacie, \\ Université de Mons (UMONS), Place du Parc 20, 7000 Mons, Belgium. \\ *Corresponding author; E-mail : hiloudio@gmail.com, 09 BP 984 Ouaga 09 Burkina Faso; \\ Tel: (226) 70734788; Fax (226) 50307242.
}

\begin{abstract}
Because of the weakness of the modern veterinarian services in Burkina Faso, ethnoveterinary medicines are frequently used to control cattle diseases like helminthe parasites. Ethnobotanic survey was performed in the central region of Burkina Faso with 22 livestock breeders on their helminthiasis management, and traditional treatments. Differences in knowledge of the disease, in treatments and in plant species used were evaluated. The relationship between informants and herbs was graphed as an interaction network. According to the survey results helminthic diseases (recognized by piloerection, lack of appetite and weight loss) are caused by water and pasture qualities. Ten single-herb preparations and 8 herbal recipes were reported. Among the 13 plant species cited, 4 are very frequently used to control helminthiasis: Mitragyna inermis (Wild.) O. Ktze, Vitellaria paradoxa Gaertn., Acacia macrostachya Rchb. ex DC., Combretum glutinosum Perr. ex DC. Ethnoveterinary practices are an economic necessity for small-scale livestock owners in rural regions of Burkina Faso. Probably, the most used species can provide, after phytochemical analysis, molecules of pharmaceutical interest. There also appears a definite need for more specific diseases diagnostics that will help to improve helminthiasis control by farmers.
\end{abstract}

(c) 2014 International Formulae Group. All rights reserved.

Keywords: Ethnoveterinary practices, Anthelminthics, breeders, Fulani, Pastoralism.

\section{INTRODUCTION}

In Burkina Faso (Figure 1), a Sahelian country situated in the heart of West Africa, livestock plays an important role in the national economy, interesting $80 \%$ of the rural population and representing $26 \%$ of export earnings and $10 \%$ of Gross Domestic Product (INSD, 2004).
Unfortunately, the quality of livestock performance has remained poor as a result of a number of animal diseases (particularly intestinal parasites and trypanosomiasis) and this has a direct effect on the economic development of the country. The treatment of gastrointestinal helminthiasis is particularly difficult because 
of polyparasitism and frequent reinfestations (Ouatarra, 2000). Moreover, different studies highlight the worldwide emergence of phenomena of resistance to the existing anthelmintics (McKellar et al. 2004; Waller et al., 2004; Geary et al., 2004) and, paradoxically, the research and development of new synthetic molecules by the pharmaceutical industry appears to be slowing.

Helminthiasis is a major concern for rural producers unable to provide good veterinarian coverage due the high cost and low availability of modern drugs. The majority of livestock owners in rural regions of Burkina Faso rely chiefly on traditional animal health practices (ethnoveterinary medicine) to control common health problems of their livestock. Most of the materia medica used in ethnoveterinary medicines is derived from plants (Mathias et al., 1996) and their use, common in the poor societies of developing nations, provides readily available low cost alternatives.

Despite the fact that ethnoveterinary medicine remains crucial for animal healthcare in most of developing countries, research effort is needed to validate the use of herbals, to elucidate their active compounds and mechanisms of action and to precise their advantages, limitations and precautions of use (Githiori et al., 2003).

This research work, undertaken in the central region of Burkina Faso, was therefore aimed at collecting the traditional knowledge of breeders and agropastoralists and to identify the ethnomedicines prescribed for the management of ruminants' intestinal parasitic diseases.

\section{MATERIALS AND METHODS}

Study area and population

Burkina Faso (Figure 1) is a landlocked country located in the heart of West Africa and wedged between six countries: Mali,
Niger, Benin, Togo, Ghana and Côte d'Ivoire. It covers an area of approximately 274000 $\mathrm{km}^{2}$. It is located inside the loop of the River Niger between $10^{\circ}$ and $15^{\circ}$ north latitude and between $2^{\circ}$ east and $5^{\circ} 30^{\prime}$ west longitude. Its capital city is Ouagadougou.

The surveys were conducted in the central region (around Ouagadougou) which has an average altitude of $350 \mathrm{~m}$. The climate is characterised by a long dry season (from October to May) and an irregular rainy season (from June to September). The homogenous and seasonal-dependant vegetal landscape is made of Parkia biglobosa (Néré), Vitellaria paradoxa (Karité), Cassia sp and Andasonia digitata ecosystems. Some species, such as Azadirachta indica (Neem) and Eucalyptus sp., have been introduced to counter desertification.

Mossi are the dominant ethnic group but many other people with different ethnic backgrounds (like Fulani) have settled in the area. Cultivation of crops like sorghum and pastoralism are the major economic activities of the local people (INSD, 2004). The Fulani people are traditionally known as livestock breeders.

\section{Sites and informants selection}

Based on information from the National Institute of Environment and Agricultural Research (INERA: Institut de l'Environnement et de la Recherche Agronomique) and the mayor office of Saaba, sampling sites for ethnobotanical data collection were selected from five sites that are located in the administrative department of Saaba (Figure 1). The market of Saaba and the Fulani camps of Saaba Boulwogodogo, Boundigui, Gampela and Gonsé. The fieldwork was performed between February and March, 2009.

A total of 22 traditional ethnoveterinary medicine practitioners were systematically chosen following the method of Martin (1995) with the help of the Saaba mayor administration, the Saaba veterinary agent and a member of the Saaba Fulani community. 


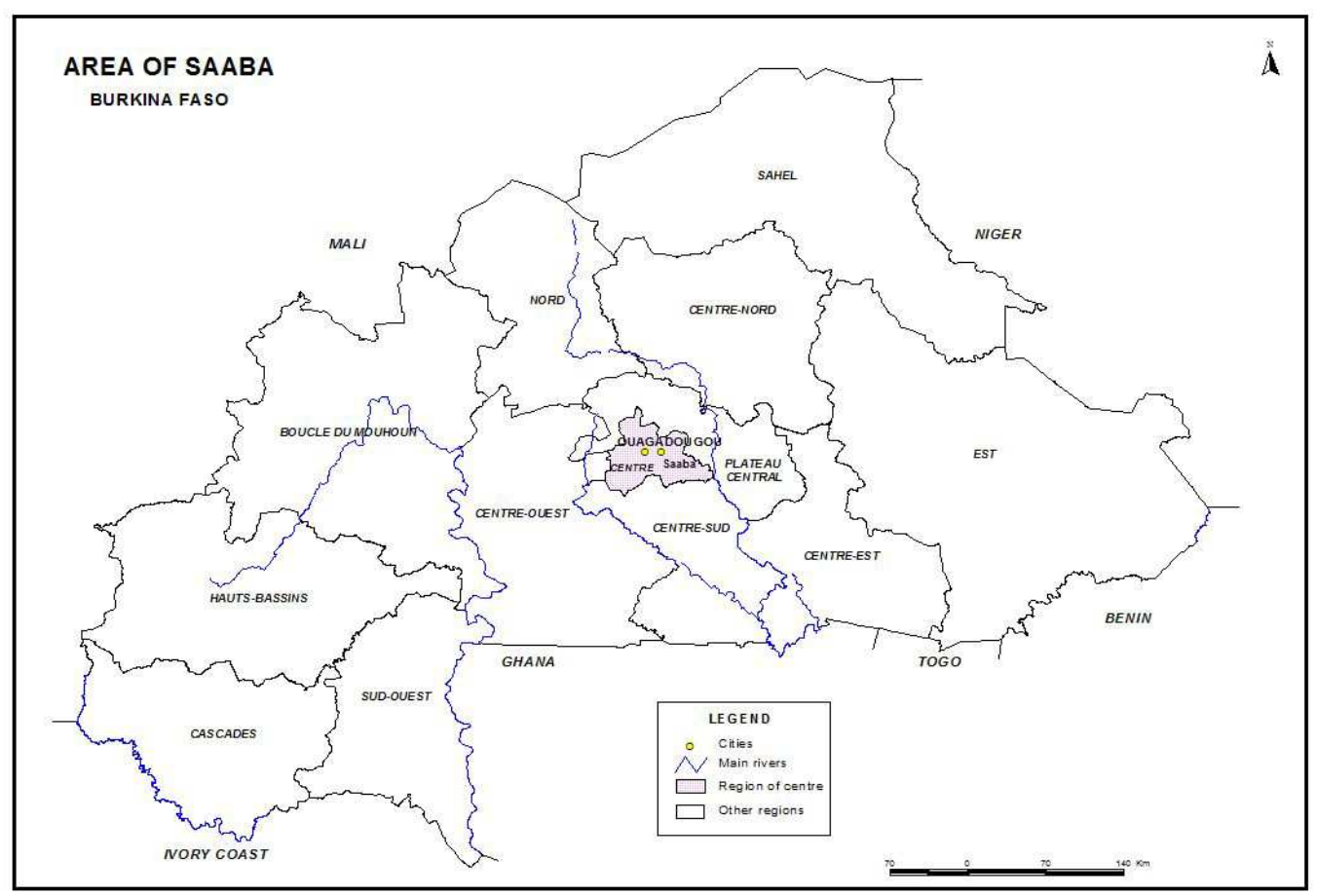

Figure 1: Study area (SABA).

\section{Semi-structured interview}

The survey was based on a questionnaire model used at the National Institute of Environment and Agricultural Researches (INERA). A confidence relationship was established with the practictioners thanks to the local veterinary agent, who is well-known and respected throughout the community.

No money was given to informers, except some small token gifts (cola nuts) to facilitate the contact. Interviews were conducted with the help of translators who were conversant with the local languages (Moore and Fulani). The mentioned plants were harvested with the help of the interviewed persons and identified by the botanist Millogo from Ouagadougou University where voucher herbarium specimens (RAPPEZ series) were deposited. The correspondences between the Fulani or Moore names and scientific names have also been verified from the literature (Von Maydell, 1990).

\section{Graphing of data}

The relationships between informants and herbs was graphed as an interaction network using the software Cytoscape 2.8.0 (http://cytoscape.org), with the layout organic (Shannon et al., 2003).

\section{RESULTS}

\section{Demographic characteristics of informants}

Twenty-two people were interviewed (Table 1), among which there were (i) 21 men and 1 woman (it was not possible to include more women due to the scarcity of female practitioners in the surveyed communities); and (ii) 20 livestock breeders of the Fulani ethnic groups and 2 agropastoralists of the Mossi ethnic group. The Fulani living in rural areas are well known to exercise the profession of breeders for many generations. 
Only 6 of the informants were literate and this in Arabic. Some informants did not know their exact age, but recorded/probable ages ranged from 32 to 77 years, most of them being over 50 (77\%). All have acquired their knowledge with their parents and village elders (mostly men), observing and accompanying them during the harvest of plants. Interviewees pick themselves the plants they use in the direct environment of the camp, near the scene of pastures and along roads leading to it, for example from bushes near water points.

\section{Knowledge of gastrointestinal parasitosis Identification of the disease}

Table 1 summarizes the information on the knowledge about gastrointestinal parasitosis. From the many symptoms cited by breeders and agro-pastoralists, the most frequent are piloerection (59\%), lack of appetite (36\%), weight loss (32\%), a phenomena of "bloat" (27\%), "pasty"stool $(27 \%)$ and diarrhea (27\%). The described symptoms are quite nonspecific and do not distinct between types of gastrointestinal disease or parasitosis. The presence of worms in the stool, the most tangible symptom, is cited by only 4 people (Informants 2, 3, 4 and 8).

\section{Causes and period of disease}

Opinions concerning the animal species most affected by gastrointestinal parasitosis are very divergent, ranging from small ruminants (sheep, goats) to cattle (Table $1)$. The main causes cited by participants include water $(82 \%)$ and pasture $(41 \%)$. Indeed, in the visited camps, the animals drink standing water (pond, dam...), a wellknown growth medium for important pests. Pastures contaminated by feces of infested animals are also an important source of contamination. Gastrointestinal parasitosis preferentially appear during the rainy season (June to October), as indicated by $68 \%$ of interviewed individuals.
However, for 5 informants (23\%), the cold dry season (November to February) is the main disease season. This discrepancy most probably arises from unreliable diagnostics that allow confusion between parasitosis and other diseases.

\section{Treatments}

Animals care

All participants treat animals presenting ascribed gastrointestinal parasitosis symptoms. Practically all of the interviewees (95\%) treat the animals by themselves, using traditional remedies; only one breeder calls the veterinary agent directly without prior treatment. However, if the illness lasts too long or if the first treatment does not work, all informants, except 2 , recourse to the veterinary agent. The reasons given to justify these choices are the high cost and low accessibility of drugs; indeed few pharmacies are found outside the major cities and there is only one veterinary officer for more than ten villages, far from each other by at least $30 \mathrm{~km}$. It should be noted that, since the advent of mobile phones, the access to veterinary agents has become easier and easier.

\section{Recommended anthelmintic plant species}

Curiously, the Mossi agro-pastoralists could not quote herbs-based recipes and claim to use only potash preparations for helminthiasis treatment; by contrast, the Fulani use a series of medicinal herbs, alone or in combination. The various plants mentioned in the survey and their use frequencies are listed in Tables 2 and 3. The most frequently cited plants are (23\%) Mitragyna inermis and Vitellaria paradoxa; and (18\%) Acacia macrostachya, Combretum glutinosum and Lannea microcarpa. Figure 2 shows the relationship between multi-herbs recipes and botanical species.

\section{Modes of uses and preparations of the plant material \\ Ten single-herb preparations and 8 herbal recipes, mostly containing 2 components, were}


reported for use in the treatment of helminthiasis (Table 2, Figure 2), giving a total of 13 different herbs. The main mode of preparation is pounding of plant organ followed by decocting or macerating in water.

Additional remedies were mentioned, based on potash, ashes or curdled milk added with salt or cow dung; scarification with a red hot sickle is also described.

Cited plants belong to 11 botanical families, the most represented families being Mimosaceae (3 species), Combretaceae (2 species) and Rubiaceae (2). The principle of polymedication is a constant in many traditional practices and relies on a general idea that "complex drugs are needed to treat complex (i.e. multifactorial) diseases" (Mukazayire et al., 2011).

It is striking to note that the traditional therapy of helminthiasis in central Burkina Faso proposes so many single-herb recipes (56\% of all herbal preparations). Mitragyna inermis is always used alone whereas Vitellaria paradoxa and Acacia macrostachya are mainly employed in mixtures.

Table 1: Details of informants and data reported on gastrointestinal parasitosis.

\begin{tabular}{|c|c|c|c|c|c|}
\hline $\begin{array}{l}\text { Informant sex, } \\
\text { age, occupation } \\
\text { and ethnic group }\end{array}$ & Observed symptoms & $\begin{array}{l}\text { Reported } \\
\text { causes }\end{array}$ & $\begin{array}{l}\text { Reported } \\
\text { period }\end{array}$ & $\begin{array}{c}\text { Animal } \\
\text { species at } \\
\text { risk }\end{array}$ & Treatment \\
\hline $\begin{array}{l}\text { M, 71, agro- } \\
\text { pastoralist, Mossi }\end{array}$ & $\begin{array}{c}\text { Lack of appetite, } \\
\text { diarrhea }\end{array}$ & $\begin{array}{l}\text { Pastures, } \\
\text { water }\end{array}$ & Rainy season & $\begin{array}{c}\text { Not } \\
\text { specified }\end{array}$ & $\begin{array}{l}\text { Himself, and } \\
\text { then if } \\
\text { necessary, the } \\
\text { veterinarian }\end{array}$ \\
\hline $\begin{array}{l}\text { M, 53, agro- } \\
\text { pastoralist, Mossi }\end{array}$ & $\begin{array}{l}\text { Slimming, pasty stool } \\
\text { with worms, lack of } \\
\text { appetite, piloerection }\end{array}$ & Water & Rainy season & $\begin{array}{c}\text { Not } \\
\text { specified }\end{array}$ & $\begin{array}{l}\text { Himself, and } \\
\text { then if } \\
\text { necessary, the } \\
\text { veterinarian }\end{array}$ \\
\hline $\begin{array}{l}\text { M, n.a., breeder, } \\
\text { Fulani }\end{array}$ & $\begin{array}{l}\text { Bloating, stool with } \\
\text { worms, piloerection }\end{array}$ & $\begin{array}{l}\text { Water, } \\
\text { habitat, } \\
\text { herbs, } \\
\text { pastures }\end{array}$ & Rainy season & $\begin{array}{c}\text { Not } \\
\text { specified }\end{array}$ & $\begin{array}{l}\text { Himself, and } \\
\text { then if } \\
\text { necessary, the } \\
\text { veterinarian }\end{array}$ \\
\hline $\begin{array}{l}\text { M, 53, breeder, } \\
\text { Fulani }\end{array}$ & $\begin{array}{c}\text { Red eyes, scales, } \\
\text { dangling earrings, stool } \\
\text { with parasites }\end{array}$ & $\begin{array}{l}\text { Standing } \\
\text { water, } \\
\text { pastures }\end{array}$ & Rainy season & $\begin{array}{c}\text { Not } \\
\text { specified }\end{array}$ & $\begin{array}{l}\text { Himself, and } \\
\text { then if } \\
\text { necessary, the } \\
\text { veterinarian }\end{array}$ \\
\hline $\begin{array}{l}\text { M, 72, breeder, } \\
\text { Fulani }\end{array}$ & $\begin{array}{l}\text { Staggering, lower } \\
\text { productivity, lack of } \\
\text { appetite, piloerection }\end{array}$ & $\begin{array}{l}\text { Water, } \\
\text { location of } \\
\text { birth }\end{array}$ & $\begin{array}{l}\text { Cold dry } \\
\text { season }\end{array}$ & $\begin{array}{c}\text { Not } \\
\text { specified }\end{array}$ & $\begin{array}{l}\text { Himself, and } \\
\text { then if } \\
\text { necessary, the } \\
\text { veterinarian }\end{array}$ \\
\hline $\begin{array}{l}\text { M, 52, breeder, } \\
\text { Fulani }\end{array}$ & Loose stools Bloating & $\begin{array}{l}\text { Food, } \\
\text { drinking } \\
\text { water }\end{array}$ & rainy season & $\begin{array}{c}\text { Not } \\
\text { specified }\end{array}$ & Himself only \\
\hline $\begin{array}{l}\text { M, } 49, \text { breeder, } \\
\text { Fulani }\end{array}$ & $\begin{array}{c}\text { Lack of appetite, bristly } \\
\text { hairs }\end{array}$ & No idea & Rainy season & Calves & $\begin{array}{c}\text { The } \\
\text { veterinarian } \\
\text { only }\end{array}$ \\
\hline
\end{tabular}




\begin{tabular}{|c|c|c|c|c|c|}
\hline $\begin{array}{l}\text { M, 32, breeder, } \\
\text { Fulani }\end{array}$ & $\begin{array}{c}\text { Bloating spots under the } \\
\text { tongue, worms in the } \\
\text { stool }\end{array}$ & $\begin{array}{l}\text { Water; } \\
\text { transmission } \\
\text { mother-child }\end{array}$ & $\begin{array}{l}\text { Cold dry } \\
\text { season }\end{array}$ & $\begin{array}{l}\text { Calves } \\
\text { sheep, } \\
\text { goats }\end{array}$ & $\begin{array}{l}\text { Himself, and } \\
\text { then if } \\
\text { necessary, the } \\
\text { veterinarian }\end{array}$ \\
\hline $\begin{array}{l}\text { M, } 44 \text {, breeder, } \\
\text { Fulani }\end{array}$ & $\begin{array}{c}\text { Weight loss, } \\
\text { constipation or diarrhea, } \\
\text { weakness, calves do } \\
\text { not suck }\end{array}$ & $\begin{array}{l}\text { Water; } \\
\text { transmission } \\
\text { mother-child }\end{array}$ & Rainy season & $\begin{array}{l}\text { Cattle, } \\
\text { sheep }\end{array}$ & $\begin{array}{l}\text { Himself, and } \\
\text { then if } \\
\text { necessary, the } \\
\text { veterinarian }\end{array}$ \\
\hline $\begin{array}{l}\text { M, 53, breeder, } \\
\text { Fulani }\end{array}$ & $\begin{array}{l}\text { Weight loss, bloating, } \\
\text { hair bristling, }\end{array}$ & $\begin{array}{l}\text { Moisture, } \\
\text { pastures, } \\
\text { breeding site }\end{array}$ & Rainy season & $\begin{array}{l}\text { Sheep, } \\
\text { goat }\end{array}$ & $\begin{array}{l}\text { Himself, and } \\
\text { then if } \\
\text { necessary, the } \\
\text { veterinarian }\end{array}$ \\
\hline
\end{tabular}

Table 1: Details of informants and data reported on gastrointestinal parasitosis (continued).

\begin{tabular}{|c|c|c|c|c|c|}
\hline $\begin{array}{l}\text { Informant } \\
\text { sex, age, } \\
\text { occupation } \\
\text { and ethnic } \\
\text { group }\end{array}$ & $\begin{array}{c}\text { Observed } \\
\text { symptoms }\end{array}$ & $\begin{array}{l}\text { Reported } \\
\text { causes }\end{array}$ & $\begin{array}{l}\text { Reported } \\
\text { period }\end{array}$ & $\begin{array}{l}\text { Animal } \\
\text { species } \\
\text { at risk }\end{array}$ & Treatment \\
\hline $\begin{array}{l}\text { M, } 72, \text { breeder, } \\
\text { Fulani }\end{array}$ & Diarrhea, bloating & Drinking water & $\begin{array}{l}\text { Cold dry } \\
\text { season }\end{array}$ & $\begin{array}{l}\text { Sheep, } \\
\text { goat }\end{array}$ & $\begin{array}{l}\text { Himself, and then } \\
\text { if necessary, the } \\
\text { veterinarian }\end{array}$ \\
\hline $\begin{array}{l}\text { M, 40, breeder, } \\
\text { Fulani }\end{array}$ & $\begin{array}{l}\text { Strange behavior, } \\
\text { dry nose, bloated } \\
\text { animal, bristly } \\
\text { hairs, lack of } \\
\text { appetite }\end{array}$ & $\begin{array}{c}\text { Pastures, } \\
\text { drinking water }\end{array}$ & $\begin{array}{l}\text { Cold dry } \\
\text { season }\end{array}$ & $\begin{array}{l}\text { Sheep, } \\
\text { goats }\end{array}$ & $\begin{array}{l}\text { Himself, and then } \\
\text { if necessary, the } \\
\text { veterinarian }\end{array}$ \\
\hline $\begin{array}{l}\text { M, } 73 \text {, breeder, } \\
\text { Fulani }\end{array}$ & $\begin{array}{l}\text { Diarrhea, weight } \\
\text { loss, constipation } \\
\text { blood in milk }\end{array}$ & $\begin{array}{l}\text { Mother-child } \\
\text { transmission, } \\
\text { lack of salty } \\
\text { feeding }\end{array}$ & $\begin{array}{l}\text { Rainy } \\
\text { season }\end{array}$ & Cattle & Himself only \\
\hline $\begin{array}{l}\text { M, } 68 \text {, breeder, } \\
\text { Fulani }\end{array}$ & $\begin{array}{l}\text { Bristly hairs, lack } \\
\text { of appetite, state of } \\
\text { stools }\end{array}$ & $\begin{array}{c}\text { Mother-child } \\
\text { transmission, } \\
\text { moisture } \\
\end{array}$ & $\begin{array}{l}\text { Cold dry } \\
\text { season }\end{array}$ & Cattle & $\begin{array}{c}\text { Himself, and then } \\
\text { if necessary, the } \\
\text { veterinarian }\end{array}$ \\
\hline $\begin{array}{l}\text { M, 33, breeder, } \\
\text { Fulani }\end{array}$ & $\begin{array}{c}\text { Bristly hairs, } \\
\text { dangling earrings, } \\
\text { lack of appetite }\end{array}$ & $\begin{array}{c}\text { Backwater, } \\
\text { moisture }\end{array}$ & $\begin{array}{l}\text { Rainy } \\
\text { season }\end{array}$ & $\begin{array}{l}\text { Sheep, } \\
\text { goat }\end{array}$ & $\begin{array}{c}\text { Himself, and then } \\
\text { if necessary, the } \\
\text { veterinarian }\end{array}$ \\
\hline $\begin{array}{l}\text { M, } 65 \text {, breeder, } \\
\text { Fulani }\end{array}$ & $\begin{array}{c}\text { Bristly hairs, } \\
\text { weight loss }\end{array}$ & Backwater & $\begin{array}{l}\text { Cold dry } \\
\text { season }\end{array}$ & Cattle & $\begin{array}{c}\text { Himself, and then } \\
\text { if necessary, the } \\
\text { veterinarian }\end{array}$ \\
\hline $\begin{array}{l}\text { M, 77, breeder, } \\
\text { Fulani }\end{array}$ & $\begin{array}{l}\text { Progressive weight } \\
\text { loss, bloating }\end{array}$ & Pastures, water & $\begin{array}{l}\text { Rainy } \\
\text { season }\end{array}$ & $\begin{array}{l}\text { Sheep, } \\
\text { goat }\end{array}$ & $\begin{array}{c}\text { Himself, and then } \\
\text { if necessary, the } \\
\text { veterinarian }\end{array}$ \\
\hline
\end{tabular}


A. HILOU et al. / Int. J. Biol. Chem. Sci. 8(5): 2207-2221, 2014

Table 1: Details of informants and data reported on gastrointestinal parasitosis (continued).

\begin{tabular}{|c|c|c|c|c|c|}
\hline $\begin{array}{l}\text { Informant sex, age, } \\
\text { occupation and ethnic group }\end{array}$ & Observed symptoms & Reported causes & $\begin{array}{c}\text { Reported } \\
\text { period }\end{array}$ & $\begin{array}{c}\text { Animal } \\
\text { species at risk }\end{array}$ & Treatment \\
\hline M, 53, breeder, Peuhl & $\begin{array}{l}\text { Lack of appetite, bristly hairs, } \\
\text { diarrhea }\end{array}$ & Water, pastures & $\begin{array}{l}\text { Rainy } \\
\text { season }\end{array}$ & Sheep, goat & $\begin{array}{l}\text { Himself, and then if } \\
\text { necessary, the } \\
\text { veterinarian }\end{array}$ \\
\hline M, 67, breeder, Fulani & $\begin{array}{l}\text { Bristly hairs, state of the } \\
\text { stools }\end{array}$ & Pastures, water & $\begin{array}{l}\text { Rainy } \\
\text { season }\end{array}$ & Sheep, goat & $\begin{array}{l}\text { Himself, and then if } \\
\text { necessary, the } \\
\text { veterinarian }\end{array}$ \\
\hline M, 53, breeder, Fulani & $\begin{array}{l}\text { Changes in hair, hair } \\
\text { bristling, diarrhea }\end{array}$ & $\begin{array}{c}\text { Sudden } \\
\text { modification of } \\
\text { feed, water } \\
\end{array}$ & $\begin{array}{l}\text { Cold dry } \\
\text { season }\end{array}$ & Cattle & $\begin{array}{l}\text { Himself, and then if } \\
\text { necessary, the } \\
\text { veterinarian }\end{array}$ \\
\hline M, 50, breeder, Fulani & $\begin{array}{l}\text { Bristly hairs, state of the } \\
\text { stools }\end{array}$ & Water, pastures & $\begin{array}{l}\text { Rainy } \\
\text { season }\end{array}$ & $\begin{array}{l}\text { Sheep, goat } \\
\text { cattle }\end{array}$ & $\begin{array}{c}\text { Himself, and then if } \\
\text { necessary, the } \\
\text { veterinarian }\end{array}$ \\
\hline F, 65, breeder, Fulani & $\begin{array}{l}\text { State of the stools, } \\
\text { progressive weight loss }\end{array}$ & Backwater & $\begin{array}{l}\text { Rainy } \\
\text { season }\end{array}$ & Calves & $\begin{array}{c}\text { Himself, and then if } \\
\text { necessary, the } \\
\text { veterinarian }\end{array}$ \\
\hline
\end{tabular}

n.a: not available 
Table 2: Anthelmintic treatments used by the informants.

\begin{tabular}{|c|c|c|c|c|}
\hline Recipe number $^{(\mathbf{a})}$ & Composition & Plant part & Recipe & Administration \\
\hline \multicolumn{5}{|l|}{$\begin{array}{l}\text { Recipe based on a } \\
\text { single herb }\end{array}$} \\
\hline 1 & $\begin{array}{c}\text { Acacia macrostachya } \text { Rchb. ex DC. } \\
\text { (Fabaceae) }\end{array}$ & Leaves & Crush and maceration & 2 oral doses, 2 consecutive days \\
\hline 2 & Albizia chevalieri Harms. (Mimosaceae) & Leaves & Crush and decoction & Unspecified \\
\hline $3(2)$ & $\begin{array}{c}\text { Boscia senegalensis (Pers) Lam. Ex Poir. } \\
\text { (Capparaceae) }\end{array}$ & Leaves & $\begin{array}{l}\text { Crush and decoction } \\
\text { or Crush and maceration }\end{array}$ & $\begin{array}{c}1 \text { oral dose or } \\
1 \text { oral dose (over } 1 \text { to } 3 \text { days) }\end{array}$ \\
\hline $4(2)$ & $\begin{array}{c}\text { Combretum glutinosum Perr. ex DC. } \\
\text { (Combretaceae) }\end{array}$ & Leaves & $\begin{array}{l}\text { Crush and decoction } \\
\text { or Crush and maceration }\end{array}$ & $\begin{array}{l}1 \text { to } 2 \text { oral administrations ( } 1 \text { or } 2 \text { days) or } \\
2 \mathrm{X} / \text { day in the nostrils and ears ( } 2 \text { days) }\end{array}$ \\
\hline 5 & Feretia apodanthera Del. (Rubiaceae) & Leaves & Crush and decoction & 1 nasal administration \\
\hline 6 & Khaya senegalensis A. Juss. (Meliaceae) & Bark & $\begin{array}{l}\text { Crush and decoction or } \\
\text { Crush and maceration }\end{array}$ & 1 oral administration on an empty stomach \\
\hline $7(2)$ & $\begin{array}{c}\text { Lannea microcarpa Engl. \& K. Krause } \\
\text { (Anacardiaceae) }\end{array}$ & Leaves & $\begin{array}{l}\text { Crush and decoction } \\
\text { or Crushand maceration }\end{array}$ & 1 oral dose \\
\hline 8 & $\begin{array}{c}\text { Mitragyna inermis (Willd.) O Ktze. } \\
\text { (Rubiaceae) }\end{array}$ & Bark & Crush and decoction & 1 or 2 oral doses ( 1 or 2 days) \\
\hline 9 & $\begin{array}{c}\text { Mitragyna inermis (Willd.) O Ktze. } \\
\text { (Rubiaceae) }\end{array}$ & Root & Crush and decoction & Unspecified \\
\hline $10(3)$ & $\begin{array}{c}\text { Mitragyna inermis (Willd.) O Ktze. } \\
\text { (Rubiaceae) }\end{array}$ & Leaves & $\begin{array}{l}\text { Crush and decoction or } \\
\text { Crush and maceration }\end{array}$ & $\begin{array}{l}2 \text { to } 3 \text { oral doses, } 3 \text { consecutive days } \\
1 \text { or more oral doses if necessary }\end{array}$ \\
\hline
\end{tabular}

(a) In brackets, number of times the recipe has been cited. 
Table 2: Anthelmintic treatments used by the informants (continued).

\begin{tabular}{|c|c|c|c|c|}
\hline Recipe number $^{(\mathbf{a})}$ & Composition & $\begin{array}{c}\text { Plant } \\
\text { part }\end{array}$ & Recipe & Administration \\
\hline \multicolumn{5}{|c|}{ Recipe based on herbs mixtures } \\
\hline \multirow[t]{2}{*}{11} & Parkia biglobosa (Jacq.) R.Br. ex G.Don (Fabaceae) & $\begin{array}{l}\text { Stem } \\
\text { bark }\end{array}$ & \multirow[t]{2}{*}{$\begin{array}{l}\text { Decoction and then mix with } \\
\text { butter }\end{array}$} & \multirow[t]{2}{*}{1 or 2 oral doses ( 1 or 2 days) } \\
\hline & Vitellaria paradoxa C.F.Gaertn. (Sapotaceae) & Root & & \\
\hline \multirow[t]{2}{*}{12} & Parkia biglobosa (Jacq.) R.Br. ex G.Don (Fabaceae) & Leaves & \multirow{2}{*}{$\begin{array}{l}\text { Crush and make a decoction of } \\
\text { the mixture of the two plants }\end{array}$} & \multirow{2}{*}{$\begin{array}{c}\text { One or several oral } \\
\text { administrations if necessary }\end{array}$} \\
\hline & Lannea microcarpa Engl. \& K. Krause (Anacardiaceae) & Leaves & & \\
\hline 13 & Vitellaria paradoxa C.F.Gaertn. (Sapotaceae) & Bark & Maceration and filtration & Unspecified \\
\hline \multirow[t]{2}{*}{14} & Acacia macrostachya Rchb. ex DC. (Fabaceae) & Leaves & \multirow{2}{*}{$\begin{array}{l}\text { Crush and maceration of the } \\
\text { mixture of the two plants }\end{array}$} & \multirow{2}{*}{$\begin{array}{c}3 \text { oral doses, } 3 \text { consecutive } \\
\text { days }\end{array}$} \\
\hline & Vitellaria paradoxa C.F.Gaertn. (Sapotaceae) & Bark & & \\
\hline \multirow[t]{2}{*}{15} & Boscia senegalensis (Pers) Lam. Ex Poir. (Capparaceae) & Leaves & \multirow{2}{*}{$\begin{array}{c}\text { Crush and decoction of the } 2 \\
\text { plants mixture }\end{array}$} & \multirow{2}{*}{$\begin{array}{l}1 \text { to } 3 \text { oral administrations with } \\
1 \text { dose/day }\end{array}$} \\
\hline & Lannea microcarpa Engl. \& K. Krause (Anacardiaceae) & Leaves & & \\
\hline 16 & Combretum micranthum G. Don (Combretaceae) & Leaves & $\begin{array}{c}\text { Crush and maceration of the } 2 \\
\text { plants mixture }\end{array}$ & $\begin{array}{l}1 \text { or more oral and nasal doses } \\
\text { if necessary }\end{array}$ \\
\hline \multirow[t]{4}{*}{17} & Acacia macrostachya Rchb. ex DC. (Fabaceae) & Leaves & \multirow{3}{*}{$\begin{array}{l}\text { Crush and maceration of the tree } \\
\text { plants }\end{array}$} & \multirow{3}{*}{$\begin{array}{c}\text { One or several oral } \\
\text { administrations if necessary }\end{array}$} \\
\hline & Maytenus senegalensis (Lam.) Excell (Celastraceae) & Leaves & & \\
\hline & Vitellaria paradoxa C.F.Gaertn. & Bark & & \\
\hline & Red hot sickle & & Markings on the flanks & Unspecified \\
\hline
\end{tabular}


Table 2: Anthelmintic treatments used by the informants (continued).

\begin{tabular}{|c|c|c|c|c|}
\hline $\begin{array}{l}\text { Recipe } \\
\text { number }\end{array}$ & Composition & Plant part & Recipe & Administration \\
\hline \multirow[t]{4}{*}{18} & Parkia biglobosa (Jacq.) R.Br. ex G.Don (Fabaceae) & Leaves & \multirow{4}{*}{$\begin{array}{l}\text { Crush together the different } \\
\text { plants, mix with water and let } \\
\text { soak }\end{array}$} & \multirow{4}{*}{$\begin{array}{l}\text { Administer orally on an empty } \\
\text { stomach, first once, then } 5 \text { days } \\
\text { and } 10 \text { days later }\end{array}$} \\
\hline & Vitellaria paradoxa C.F.Gaertn. (Sapotaceae) & Leaves & & \\
\hline & Acacia macrostachya Rchb. ex DC. (Fabaceae) & Leaves, root & & \\
\hline & $\begin{array}{lllll}\begin{array}{l}\text { Securinega virosa } \\
\text { (Phyllanthaceae) }\end{array} & \text { (Roxb. } & \text { ex } & \text { Willd. }) & \text { Baill. }\end{array}$ & Leaves, root & & \\
\hline \multicolumn{5}{|c|}{ Methods not based on herbs } \\
\hline 19 & Potash & & Crush and mix with water & 1 oral administration \\
\hline \multirow[t]{2}{*}{20} & Curdled milk + salt & & Mix with water & 1 oral administration \\
\hline & Ash & & & \\
\hline 21 & Curdled milk + cow dung & & $\begin{array}{l}\text { Curdle milk and then mix it with } \\
\text { cow dung }\end{array}$ & 1 or more oral doses if necessary \\
\hline $22(2)$ & Red hot Sickle & & $\begin{array}{l}\text { Markings, from the hump to the } \\
\text { tail of the animalor Draw a cross } \\
\text { on the forehead of the animal }\end{array}$ & Unspecified \\
\hline
\end{tabular}


Table 3: Plants use frequency.

\begin{tabular}{|c|c|c|c|c|}
\hline Latin binomial & Fulani Name & Mooré Name & $\begin{array}{c}\text { Number of citing } \\
\text { informants }\end{array}$ & $\begin{array}{c}\text { Frequency } \\
(\%)\end{array}$ \\
\hline Acacia macrostachya Rchb. ex DC. (Fabaceae) & Kedi, tchidi & Zamenega, guenbaogo & $4 / 22$ & 18.2 \\
\hline Albizia chevalieri Harms. (Mimosaceae) & Gondogahidgariahi & Dosendouaga & $1 / 22$ & 4.5 \\
\hline Boscia senegalensis (Pers) Lam. Ex Poir. (Capparaceae) & Djigilli, gegilli & Lanwetga, nabediga & $3 / 22$ & 13.6 \\
\hline Combretum glutinosum Perr. ex DC. (Combretaceae) & Dooki, ookai & Dandegha, koagenga & $4 / 22$ & 18.2 \\
\hline Combretum micranthum G. Don (Combretaceae) & Goungumi, gougumi & Ramdega, randiga & $1 / 22$ & 4.5 \\
\hline Feretia apodanthera Del. (Rubiaceae) & Ibbi, obbi, boraouhi & Borouhi, fininga & $1 / 22$ & 4.5 \\
\hline Khaya senegalensis A. Juss. (Meliaceae) & Kahi, kail, cail & Kuka & $2 / 22$ & 9.1 \\
\hline Lannea microcarpa Engl. \& K. Krause (Anacardiaceae) & Falfami, peguhi & Sabtoulouga, siibiga & $4 / 22$ & 18.2 \\
\hline Maytenus senegalensis (Lam.) Excell (Celastraceae) & Giyalgoti, yengotehi & & $1 / 22$ & 4.5 \\
\hline Mitragyna inermis (Willd.) O Ktze. (Rubiaceae) & Kooli, kauli & Yilga, hiliga, jilega & $5 / 22$ & 22.7 \\
\hline Parkia biglobosa (Jacq.) R.Br. ex G.Don (Fabaceae) & Nere, narehi, nerehi & Doaaga, teenga & $3 / 22$ & 13.6 \\
\hline Securinega virosa (Roxb. ex Willd.) Baill. (Phyllanthaceae) & Sugurlaagahi, boboli & Boufobou, punpunga & $1 / 22$ & 4.5 \\
\hline Vitellaria paradoxa C.F.Gaertn. (Sapotaceae) & & & $5 / 22$ & 23 \\
\hline
\end{tabular}




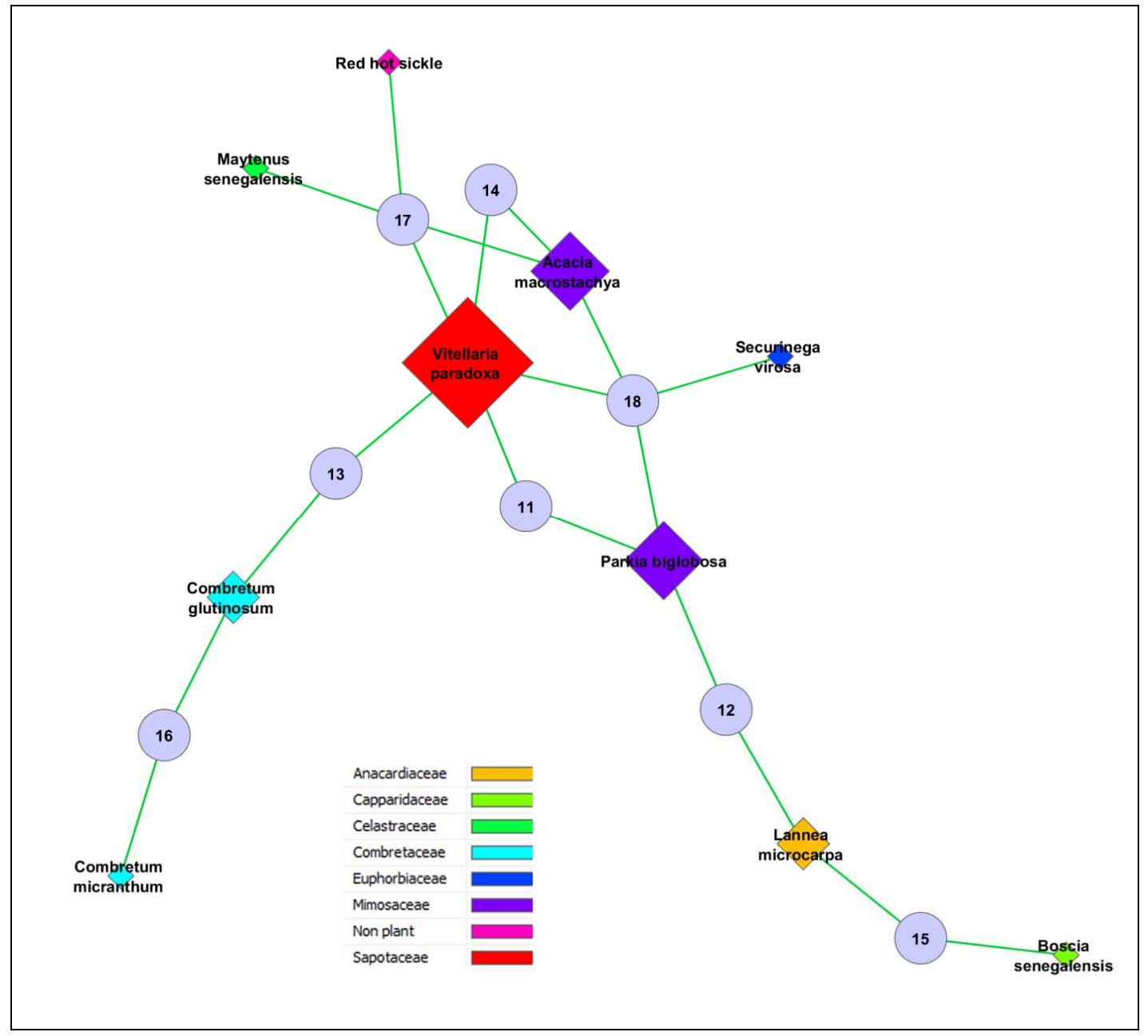

Figure 2: Relationships between multi-herbal recipes and medicinal plants. Recipes are represented as circles, medicinal plants as diamonds; the size of the diamond is proportional to the frequency of citation in multi-herbal recipes. The colors of diamonds correspond to the botanical family of the plant. (Shannon et al., 2003; Mukazayire et al, 2011).

\section{DISCUSSION}

The results of this survey have shown a more or less frequent use of certain plant species to treat heminthiases in this region. Through a review of the literature, one can compare the uses listed here to pharmacological studies reports.

Regarding Combretum glutinosum Perr. Ex.DC., we could not find literature study on their anthelmintic activity. However, we were able to identify a study showing different biological activities, including the anthelmintic activity of other 20 species of the genus Combretum. (McGaw et al., 2001).

Acacia Macrostachya Rchb. ex DC.: We could not identify any study on the anthelmintic activity of Acacia macrostachya. Nonetheless, there are several studies showing an anthelmintic activity of other species of Acacia as Acacia molissima (Minho et al., 2008). 
Albizia chevalieri Harms.: No studies on the anthelmintic activity of Albizia chevalieri Harms was found. However, several studies have shown an anthelmintic activity of Albizia anthelminthica (Githiori et al., 2003; Grade et al., 2008).

Boscia senegalensis (Pers.) Lam. ex Poir. : No study on the anthelmintic activity of Boscia senengalensis or another plant of the genus Boscia has been identified. However, an ethnobotanical survey in Niger on the traditional treatment of camels' diseases indicates that Boscia senegalensis is used for treatment of chronic helminth infections. The leaves of $B$. senegalensis crushed and mixed with chewing tobacco and salt or boiled are used mixed to Cucumis prophetarum. Another preparation method is to grind the plant and soak in the urine of sheep for $24 \mathrm{~h}$. The filtered liquid is administered either orally or nasally for 3 days (Antoine-Moussieux N., 2007).

In our survey, the leaves of $B$. senegalensis are also used after decoction or maceration and pounding.

Khaya senegalensis (Desr.) A. Juss.: A study showed that the bark of $K$. senegalensis possesses anthelmintic activity (Ademola et al., 2004). It is interesting to note that it is also the use of the bark of the plant that occurred during our investigation.

Lannea microcarpa Engl. et $\mathrm{K}$. Krause: No studies on the anthelmintic activity of L. microcarpa or another plant of the genus Lannea could be found.

Mitragyna inermis (Wild.) O. Ktze. : Antibacterial and muscle-relaxant activities of the plant could be detected (Sy et al., 2004; Asase et al., 2008) but there are no studies pertaining to the anthelmintic activity of $M$. inermis or another plant of the genus Mitragyna.

Vitellaria paradoxa C.F. Gaertn. : No studies on the anthelmintic activity of
Vitellaria paradoxa or another plant of this genus were identified.

\section{Conclusion}

This ethnobotanic survey results show that there is much traditional knowledge concerning cattle anthelmintic diseases and their traditional treatment within the Fulani and Mossi communities in central Burkina Faso. They can identify many helminthic diseases. They can treat most of these diseases using 13 herbal plants and other materials and methods. These treatments and practices reported in this study need to be validated in order to identify those which can be used for agriculture development in rural context of Africa. Mitragyna inermis, Vitellaria paradoxa, Acacia macrostachya, Combretum glutinosum, four of the more frequently used species can be good candidates for efficacy, bioactive principles and standardisation investigations.

Even if these plants may have bioactive principle, there is also a need to improve the livestock breeders' diagnosis capacitation by use of a best specific symptoms (presence of worms in the stool, for example), because there are many other ailments which can cause diarrhea. The improvement of the livestock conditions, including the drilling of wells in the vicinity of the Fulani's camps can also contribute to the decrease of animal diseases.

\section{ACKNOWLEDGEMENTS}

The authors would like to thank Le Conseil Interuniversitaire de la Communauté Francophone de Belgique, Commmission Universitaire pour le Développement for financing Florence RAPPEZ during her master in pharmacy program. The cattle breeders that participated in the ethnobotanical survey, Seydou Barry 
(SABA veterinary agent) and Hamadou Djané (Moore and Fulani translator) are also thanked for their support, help and great hospitality. The laboratory analyses were financed by a IFS grant (Project E/4627-1).

\section{REFERENCES}

Ademola IO, Fagbemi BO, Idowu SO. 2004. Evaluation of the anthelmintic activity of Khaya senegalensis extract against gastrointestinal nematodes of sheep: in vitro and in vivo studies. Veterin. Parasitol., 122: 151-164.

Antoine-Moussiaux N, Faye B, Vias GF. 2007. Tuareg ethnoveterinary treatments of camel diseases in Agadez area (Niger). Trop. Anim. Health. Prod., 39: 83-89.

Asase A, Kokubun T, Grayer RJ, Kite G, Simmonds MSJ, Yeboah AAO, Odamtten GT. 2008. Chemical constituents and antimicrobial activity of medicinal plants from Ghana: Cassia sieberiana, Haematostaphis barteri, Mitragyna inermis and Pseudocedrela kotschyi. Phytother. Res., 22: 10131016.

Geary TG, Conder GA, Bishop B. 2004. The changing landscape of antiparasitic drug discovery for veterinary medicine. Trends in Parasitol., 20(10): 449-455.

Githiori JB, Höglund J, Waller PJ, LeydenBaker R. 2003. The anthelmintic efficacy of the plant, Albizia anthelmintica, against the nematode parasites Haemonchus contortus of sheep and Heligmosomoides polygyrus of mice. Veterin. Parasitol., 116: 23-34.

Grade JT, Arble BL, Weladji RB, Van Damme P. 2008. Anthelmintic efficacy and dose determination of Albizia anthelmintica against gastrointestinal nematodes in naturally infected ugandan sheep. Veterin. Parasitol., 157: 267-274.

INSD (Institut National de Statistiques et de la Démographie). 2004. Statistiques sur les conditions de vie des ménages. Ministère de l'économie et des finances $\mathrm{du}$ Burkina Faso, Ouagadougou.

Martin GJ. 1995. Ethnobotany: a 'People and Plants' Conservation Manual. Chapman and Hall: London; 268.

Mathias E, McCorkle M, Van Veen TWC. 1996. Introduction to Ethnoveterinary research and development. In Ethnoveterinary Research and Development, McCorkle CM, Mathias E, Schillhorn van Veen TW, (eds). Intermediate Technology Publications: London.

McGaw LJ, Rabe T, Sparg SG, Jäger AK, Eloff JN, van Staden J. 2001. An investigation on the biological activity of Combretum species. J. Ethnopharmacol., 75: 45-50.

Mckellar QA, Jackson F. 2004. Veterinary anthelmintics: old and new. Trends in Parasitol., 20(10): 456-461.

Minho MA, Bueno ICS, Louvandini $\mathrm{H}$, Jackson F, Gennari SM, Abdalla AL. 2008. Effect of Acacia molissima tannin extract on the control of gastrointestinal parasites in sheep. Anim. Feed Sci. Technol., 147: 72181.

Mukazayire M-J, Minani V, Ruffo CK, Bizuru E, Stévigny C, Duez P. 2011. Traditional phytotherapy remedies used in Southern Rwanda for the treatment of liver diseases. $J$. Ethnopharmacol., 138(2): 415-431. 
Ouatarra O.2000. Etude de l'activité pharmacologique des amandes de Balanites aegyptica (L) Del (Balanitaceae): Propriétés anthelminthiques et molluscicides. Thèse de pharmacie, Université de Ouagadougou.

Shannon P, Markiel A, Ozier O, Baliga N, Wang J, Ramage D, Amin N, Schwikowski B, Ideker T. 2003. Cytoscape: a software environment for integrated models of biomolecular interaction networks. Genome Research, 13: 2498-2504.
Sy GY, Sarr A, Dieye AM, Faye B. 2004. Myorelaxant and antispasmodic effects of the aqueous extract of Mitragyna inermis barks on Wistar rat ileum. Fitoterapia, 75 (5): 447-450.

Von Maydell HJ. 1990. Trees and Shrubs of the Sahel, their Characteristics and Uses, GTZ (ed). Verlag Josef Margraf: Weikersheim.

Waller PJ, Thamsborg SM. 2004. Nematode control in 'green' ruminant production systems. Trends in Parasitol., 20(10): 493-497. 